\title{
PPP COM INTEGRAÇÃO DE DADOS GPS/GLONASS: FUNDAMENTOS ENVOLVIDOS E ANÁLISE DE ACURÁCIA
}

\author{
PPP with GPS/GLONASS data Integration: Fundamentals Involved and \\ Accuracy Assessment
}

\author{
Emanoel Gomes de Sousa Silva ${ }^{1}$ \\ Haroldo Antonio Marques ${ }^{1}$
}

1 Universidade Federal de Pernambuco - UFPE.Centro de Tecnologia e Geociências/ Departamento de
Engenharia Cartográfica, Recife, PE. Programa de Pós-Graduação em Ciências Geodésicas e
Tecnologias da Geoinformação. Email:emanoelg3@gmail.com; haroldoh2o@gmail.com.

\section{Resumo}

Atualmente o GNSS possui o GPS e GLONASS operacionais, o que possibilita a integração destes sistemas no posicionamento geodésico. A integração de dados GPS/GLONASS fornece melhor geometria e maior confiabilidade na estimativa dos parâmetros envolvidos. Ao aplicar a integração no posicionamento é necessário realizar a compatibilização do sistema de tempo e também do Sistema Geodésico de Referência no caso da utilização de órbitas transmitidas. Em termos de posicionamento geodésico especial atenção tem sido dada ultimamente para o método PPP, o qual requer um único receptor no nível de usuário, órbitas e correções precisas de relógios dos satélites, além da modelagem matemática que permita corrigir os diversos efeitos envolvidos com a propagação dos sinais GNSS e efeitos geodinâmicos que afetam a estação. Neste artigo apresentam-se os modelos matemáticos do ajustamento no modo PPP com integração de dados GPS/GLONASS, além da análise de acurácia posicional envolvendo dados coletados por receptores em estações brasileiras. Os processamentos de dados foram realizados a partir do software GPSPPP do NRCAN com soluções diárias e a geração de série temporal anual. As análises foram realizadas em termos de Erro Médio Quadrático diário e foram observadas melhorias de até $25 \%$ na estimativa da posição ao aplicar os dados GPS/GLONASS no PPP ao invés de somente dados GPS.

Palavras-chave: GNSS; Integração GPS e GLONASS; PPP.

\begin{abstract}
:
Currently GNSS has GPS and GLONASS operating, which enables the integration of these systems in geodetic positioning. The integration of GPS/GLONASS data provides better geometry and greater reliability in the estimation of the involved parameters. By applying the integration in the positioning it is necessary to perform the compatibility of the time system and also of the Geodetic Reference System, in case of applying broadcasted orbits. In terms of geodetic positioning special attention has been given nowadays for the PPP method that requires
\end{abstract}


a single receiver at the user level, precise orbit and clock corrections for satellites, as well as mathematical modeling capable to correct for several effects involved in the GNSS signals propagation and the geodynamic effects in the station. In this paper it will be presented the adjustment mathematical model for PPP with integration of GPS/GLONASS data, as well as analysis of positional accuracy involving data collected by receivers in Brazilian stations. The data processing was carried out by using the GPSPPP NRCan software considering daily solutions with generation of annual time series. Analyses were performed in terms of daily Root Mean Square and improvements were observed reaching up to $25 \%$ in the position estimation when applying GPS/GLONASS in the PPP instead of only GPS data.

Keywords: GNSS; GPS and GLONASS integration; PPP.

\section{Introdução}

O posicionamento geodésico atualmente é realizado em sua maioria a partir do GNSS (Global Navigation Satellite System), podendo-se destacar o GPS (Global Positioning System) e o GLONASS (GLObal'naya NAvigatsionnaya Sputnikkovaya Sistema), os quais se encontram atualmente em sua forma operacional. Outros sistemas, tais como o GALILEO (European Global Navigation Satellite System) e o COMPASS/BeiDou (CNSS - Compass Navigation Satellite System) se encontram em fase de desenvolvimento. Dentre os sistemas de navegação, o GPS ainda é o mais utilizado na atualidade considerando que este tem sido aplicado como sistema de navegação em sua forma operacional completa por aproximadamente três décadas. Contudo, o GLONASS atualmente também se encontra em sua forma totalmente operacional com uma constelação de satélites completa.

Atualmente, o GPS e o GLONASS passam por uma fase de modernização, a qual envolve o segmento espacial e o de controle. A modernização do GLONASS contempla a transmissão de sinais com base na técnica CDMA (Code Division Multiple Access) em três portadoras e a modernização do GPS envolve a inclusão do novo código civil na portadora L2 (L2C), o código civil na L1 (L1C), além da nova portadora L5 e sinais militares. (Hofmann-Wellenhof et al., 2008; ICD-GLONASS, 2008). O sinal L2C e a portadora L5 já estão disponíveis a partir dos satélites GPS do bloco IIR e IIF. Estes fatores favorecem a interoperabilidade dos sistemas de navegação abrindo oportunidades seja para aplicações de ordem prática ou de pesquisa científica. Em relação ao posicionamento destaca-se método PPP (Posicionamento por Ponto Preciso), o qual tem sido investigado pela comunidade cientifica nos últimos anos. Este método quando aplicado em sua forma convencional requer o uso de órbitas e correções precisas de relógios dos satélites, aplicação da observável livre da ionosfera (ion-free) para a pseudodistância e fase da onda portadora, além da modelagem matemática dos efeitos envolvidos visando obtenção de coordenadas com acurácia centimétrica. A estimativa das coordenadas pode ser no modo PPP pós-processado ou em quase tempo real (latência de poucos segundos) (Marques et al., 2014). Os efeitos envolvidos estão relacionados principalmente com a propagação dos sinais GNSS, como por exemplo, aqueles provocados pela ionosfera e troposfera, multicaminho, erro da órbita, erros de relógios do receptor e satélites, efeitos de relatividade, atraso de hardware, centro de fase da antena do satélite e do receptor, entre outros. (Seeber, 2003; Hofmann-Wellenhof et al., 2008; Monico, 2008).

Neste trabalho, apresentam-se os modelos matemáticos envolvidos com integração GPS/GLONASS no PPP e análise da acurácia posicional envolvendo dados GNSS coletados em 
estações da RBMC (Rede Brasileira de Monitoramento Contínuo dos sistemas GNSS). Para análise de acurácia foram realizados processamentos de dados no modo PPP com o software GPSPPP do NRCAN (Natural Resources Canada), onde, primeiramente foram utilizadas somente medidas GPS e em seguida medidas GPS/GLONASS com soluções diárias e a geração de série temporal anual. As coordenadas estimadas, seja aplicando somente GPS ou GPS/GONASS, foram comparadas com as coordenadas conhecidas das estações e análises foram realizadas em termos de Erro Médio Quadrático (EMQ). Análise da geometria posicional também foi realizada e, em geral, verificam-se melhorias de até $25 \%$ na acurácia posicional ao aplicar os dados GPS/GLONAS no PPP ao invés de somente GPS.

\section{Modelo Matemático do PPP com integração GPS/GLONASS}

Os satélites GPS transmitem sinais gerados a partir de uma frequência fundamental $\left(\mathrm{f}_{0}\right)$ de 10,23 $\mathrm{MHz}$, cuja multiplicação por números inteiros, 154, 120 e 115 permite formar a frequência de cada uma das três portadoras denominadas de L1 (1575,42 MHz), L2 (1227,60 MHz) e L5 $(1176,45 \mathrm{MHz})$. Os sinais são transmitidos com base na técnica CDMA, no qual todos os satélites podem transmitir numa mesma frequência e serem identificados pelo seu código PRN (PseudoRandom Noise). Diferentemente do GPS, cada satélite GLONASS transmite sinais em frequências específicas. Desta forma, a identificação do satélite se dá pela frequência do sinal, técnica denominada FDMA (Frequency Division Multiple Access) (Seeber, 2003; Leick, 2004; Monico, 2008). Contudo, o GLONASS já possui atualmente dois satélites (GLONASS-K) transmitindo dados com base na técnica CDMA. As frequências em cada satélite GLONASS são definidas por (Roosbach, 2001; Hofmann-Wellenhof et al., 2008):

$$
\begin{aligned}
& f_{1 k}=f_{1}+\Delta f_{1} \cdot k=1602,000+(0,5625 \cdot k) M H z \\
& f_{2 k}=f_{2}+\Delta f_{2} \cdot k=1246,000+(0,4375 \cdot k) M H z \\
& f_{3 k}=f_{3}+\Delta f_{3} \cdot k=1204,704+(0,4230 \cdot k) M H z
\end{aligned}
$$

As frequências $f_{1}, f_{2}$ e $f_{3}$ estão, respectivamente, associadas às portadoras L1, L2 e L3. O valor de $\mathrm{k}$ diferencia os canais de frequência. Os termos $\Delta \mathrm{f}_{1}, \Delta \mathrm{f}_{2}$ e $\Delta \mathrm{f}_{3}$ são fatores que denotam incrementos na frequência de sinais dos satélites em dois canais adjacentes (Hofmann-Wellenhof et al., 2008). As equações de pseudodistância (PD) e de fase da onda portadora para o GPS e GLONASS em unidades de metro podem ser escritas como (Seebeer, 2003; Monico, 2008):

$$
\begin{aligned}
& P D_{L_{i}}^{G}=\rho^{G}+c\left(d t_{r \theta c}^{G}-d t_{s a t}^{G}\right)+I_{L_{i}}^{G}+T^{G}+O r b^{G}+d m^{G}+v_{P D^{G}}^{G} \\
& \lambda_{L_{i}}^{G} \Phi_{L_{i}}^{G}=\rho^{G}+c\left(d t_{r \theta c}^{G}-d t_{s a t}^{G}\right)-I_{L_{i}}^{G}+T^{G}+O r b^{G}+d m^{G}+\lambda_{L_{i}}^{G} N_{L_{i}}^{G}+v_{\phi_{L_{i}}^{G}} \\
& P D_{L_{i}}^{R}=\rho^{R}+c\left(d t_{r \theta c}^{R}-d t_{s a t}^{R}\right)+I_{L_{i}}^{R}+T^{R}+O r b^{R}+d m^{R}+v_{P D_{L_{i}}^{R}} \\
& \lambda_{I}^{R} \Phi_{L_{i}}^{R}=\rho^{R}+c\left(d t_{r e c}^{R}-d t_{s a t}^{R}\right)-I_{L_{i}}^{R}+T^{R}+O r b^{R}+d m^{R}+\lambda_{I}^{R} N_{L_{i}}^{R}+v_{\phi_{L_{i}}^{R}} .
\end{aligned}
$$

Nas Equações 4 a 7, os símbolos G e R são usados para representar respectivamente o GPS e o GLONASS. A distância geométrica entre o satélite e o receptor é representada por $\rho$ e os erros dos relógios do receptor e do satélite são representados, respectivamente, por $d t_{\text {rec }}$ e $d t_{\text {sat }}$. A ambiguidade da portadora é denotada por $N_{L_{i}}$ e os erros da ionosfera, troposfera, órbita e 
multicaminho são representados, respectivamente, por $I_{L i}$ e $T$, Orb e dm. Os termos $v_{P D^{G}}, v_{\Phi_{L_{i}}^{G} \text {, }}$ $v_{P D_{L_{i}}^{R}}, v_{\Phi_{L_{i}}^{R}}$ representam erros aleatórios ou não modelados. $\mathrm{O}$ subscrito $\mathrm{L}_{\mathrm{i}}$ se refere à frequência da portadora.

No caso do PPP, a posição e o erro do relógio do satélite são, geralmente, obtidos a partir das efemérides precisas e arquivos de correções de relógios, os quais atualmente são disponibilizados pelos centros do IGS tanto para o GPS quanto para o GLONASS. Neste caso, o SGR (Sistema Geodésico de Referência) ao qual a órbita está vinculada é um dos ITRFs (International Reference Frame), atualmente o ITRF2008. No caso da utilização de efemérides transmitidas, é importante destacar que é necessária a contabilização do SGR, uma vez que a órbita transmitida do GPS é dada no WGS84 e a do GLONASS no PZ90. Enquanto a órbita do GPS é disponibilizada em forma de elementos Keplerianos, a do GLONASS é dada em forma de coordenadas cartesianas, velocidades e acelerações, requerendo um processo de integração numérica do modelo dinâmico de órbita (Stewart; Tsakiri, 1998).

$\mathrm{O}$ erro do relógio do receptor é calculado como a diferença entre o instante de recepção $\left(\mathrm{t}_{\mathrm{r}}\right)$ no receptor e sistema de tempo GPS. O mesmo ocorre para o erro do relógio no caso do GLONASS, porém, este é obtido com base na diferença entre o tempo de recepção no receptor em relação ao sistema de tempo GLONASS (ver Equações 6 e 7). Desta forma, o erro do relógio do receptor pode ser representado por (Cai; Gao, 2007):

$$
d t_{r e c}=t-t_{s y s}
$$

O termo $t_{\text {sys }}$ denota o sistema de tempo GPS ( $\mathrm{T}_{\mathrm{GPS}}$ ) para observações GPS ou o sistema de tempo

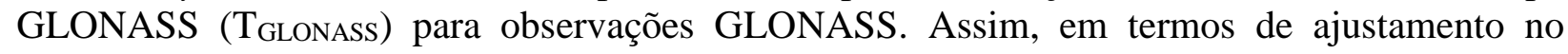
modo PPP com integração GPS/GLONASS, comparecem dois parâmetros relacionados ao erro de relógio do receptor. Por esta razão, utiliza-se a estratégia de estimar o atraso entre os sistemas de tempo GPS e GLONASS, o qual é denotado por dt $\mathrm{sys}_{\text {sys }}$ Fazendo uso da Equação 8 para o caso do erro do relógio do receptor em relação ao GLONASS, tem-se (Cai; Gao, 2007):

$$
\begin{aligned}
& d t_{r e c}^{R}=t_{r}-t_{\text {GLONASS }}=\underbrace{t_{r}-t_{G P S}}_{d t_{r e c}^{G}}+\underbrace{t_{G P S}-t_{\text {GLONASS }}}_{d t_{\text {sys }}} \\
& \Rightarrow d t_{r e c}^{R}=d t_{r e c}^{G}-d t_{s y s}
\end{aligned}
$$

Verifica-se na Equação 9 que o erro do relógio do receptor em relação ao tempo GLONASS $\left(d t_{r e c}^{R}\right)$ é calculado em função do erro do relógio em relação ao tempo GPS ( $d t_{r e c}^{G}$ ) e em relação ao atraso entre os sistemas de tempo GPS e GLONASS $\left(d t_{s y s}\right)$. Aplicando a Equação 9 nas Equações 6 e 7, tem-se:

$$
\begin{aligned}
& P D_{r}^{s}=\rho^{G}+c(\underbrace{d t_{r e c}^{G}+d t_{s y s}}_{d t_{r e c}^{R}}-d t_{s a t}^{R})+I_{L i}^{R}+T^{R}+O r b^{R}+d m^{R}+v_{P D^{R}}^{R} \\
& \lambda_{L i}^{R} \Phi_{L i}^{R}=\rho^{R}+c(\underbrace{d t_{r e c}^{G}+d t_{s y s}}_{d t_{r e c}^{R}}-d t_{s a t}^{R})-I_{L i}^{R}+T^{R}+O r b^{R}+d m^{R}+\lambda_{L i}^{R} N_{L i}^{R}+v_{\Phi_{L i}^{R}}^{R}
\end{aligned}
$$

Para usuários com receptores de dupla frequência, os efeitos ionosféricos de primeira ordem podem ser praticamente eliminados através da combinação ion-free (livre da ionosfera). Os 
efeitos ionosféricos de ordem superior podem ser corrigidos conforme descrito em Marques et al., (2011). As equações da combinação ion-free para a pseudodistância e fase do GPS e GLONASS são dadas por:

$$
\begin{aligned}
& P D_{I F}^{G}=\frac{\left(f_{G L 1}^{2} P D_{L 1}^{G}-f_{G L 2}^{2} P D_{L 2}^{G}\right)}{\left(f_{G L 1}^{2}-f_{G L 2}^{2}\right)}=\rho^{G}+c\left(d t_{r e c}^{G}-d t_{s a t}^{G}\right)+m_{f} T_{Z W}+O r b^{G}+d m^{G}+v_{P D_{I F}^{G}} \\
& \lambda_{I F}^{G} \Phi_{I F}^{G}=\frac{\left(f_{G L 1}^{2} \Phi_{L 1}^{G}-f_{G L 2}^{2} \Phi_{L 2}^{G}\right)}{\left(f_{G L 1}^{2}-f_{G L 2}^{2}\right)}=\rho^{G}+c\left(d t_{r e c}^{G}-d t_{s a t}^{G}\right)+m_{f} T_{Z W}+O r b^{G}+d m^{G}+\lambda_{I F}^{G} N_{I F}^{G}+v_{\Phi_{I F}^{G}} \\
& P D_{I F}^{R}=\frac{\left(f_{R L 1}^{2} P D_{L 1}^{R}-f_{R L 2}^{2} P D_{L 2}^{R}\right)}{\left(f_{R L 1}^{2}-f_{R L 2}^{2}\right)}=\rho^{R}+c\left(d t_{r e c}^{G}+d t_{s y s}-d t_{s a t}^{R}\right)+m_{f} T_{Z W}+O r b^{R}+d m^{R}+v_{P D_{I F}^{R}} \\
& \lambda_{I F}^{R} \Phi_{I F}^{R}=\frac{\left(f_{R L 1}^{2} \Phi_{L 1}^{R}-f_{R L 2}^{2} \Phi_{L 2}^{R}\right)}{\left(f_{R L 1}^{2}-f_{R L 2}^{2}\right)}=\rho^{R}+c\left(d t_{r e c}^{G}+d t_{s y s}-d t_{s a t}^{R}\right)+m_{f} T_{Z W}+O r b^{R}+d m^{R}+\lambda_{I F}^{R} N_{I F}^{R}+v_{\Phi_{I F}^{R}}
\end{aligned}
$$

Nas Equações 12 a 15 têm-se como parâmetros as coordenadas da estação, erro do relógio do receptor em relação ao tempo GPS, diferença do sistema de tempo $\left(d t_{s y s}\right)$, as ambiguidades da fase e a componente úmida da troposfera $(T z w)$ na direção zenital sendo que $m_{f}$ representa sua função de mapeamento (Monico, 2008; Marques et al., 2014, Sapucci, 2001). As órbitas e erros de relógios dos satélites advêm das efemérides precisas e outros erros envolvidos devem ser modelados. Desta forma, as equações de pseudodistância e fase em sua forma linearizada (Gemael, 1994) podem ser escritas como:

$$
\begin{aligned}
& E\left\{\Delta P D_{I F}^{G}\right\}=-\frac{X^{5} G-X_{r e c}^{0}}{(\rho G)^{0}} \Delta X_{r e c}-\frac{Y^{5} G-Y_{r e c}^{0}}{\left(\rho_{G}\right)^{0}} \Delta Y_{r e c}-\frac{z^{5} G-z_{r e c}^{0}}{(\rho G)^{\circ}} \Delta Z_{r e c}+c\left(c d t_{r e c}^{G}\right)+m_{f} T z W \\
& E\left\{\Delta \phi_{I F}^{G}\right\}=-\frac{X^{S} G-X_{r e c}^{0}}{(\rho G)^{\circ}} \Delta X_{r e c}-\frac{Y^{S} G-Y_{r e c}^{0}}{(\rho G)^{\circ}} \Delta Y_{r e c}-\frac{z^{S G-z_{r e c}^{0}}}{(\rho G)^{\circ}} \Delta Z_{r e c}+c\left(c d t_{r e c}^{G}\right)+m_{f} T z W+\lambda_{I F}^{G} N_{I F}^{G} \\
& E\left\{\Delta P D_{I F}^{R}\right\}=-\frac{X^{S}-X_{r e c}^{\rho}}{\left(\rho_{R}\right)^{0}} \Delta X_{r e c}-\frac{Y^{S_{R}-Y_{r e c}^{0}}}{\left(\rho_{R}\right)^{0}} \Delta Y_{r e c}-\frac{z^{S_{R}-z_{r e c}^{0}}}{\left(\rho_{R}\right)^{0}} \Delta Z_{r e c}+c\left(c d t_{r e c}^{R}\right)+m_{f} T z w \\
& E\left\{\Delta \phi_{I F}^{R}\right\}=-\frac{X^{S_{R}-X_{r e c}^{0}}}{\left(\rho_{R}\right)^{\circ}} \Delta X_{r e c}-\frac{Y_{R}^{S_{R}-Y_{r e c}^{0}}}{\left(\rho_{R}\right)^{\circ}} \Delta Y_{r e c}-\frac{z^{S_{R}-z_{r e c}^{0}}}{\left(\rho_{R}\right)^{0}} \Delta Z_{r e c}+c\left(c d t_{r e c}^{R}\right)+m_{f} T z W+\lambda_{I F}^{R} N_{I F}^{R}
\end{aligned}
$$

Onde o termo $\Delta$ é usado para representar as diferenças entre valores observados e calculados, ou seja, o vetor $\mathrm{L} X^{S}$. Os termos $X^{S_{i}}, Y^{S_{i}}$ e $Z^{S_{i}}$ representam as coordenadas dos satélites, seja GPS ou GLONASS ( $\mathrm{i}=\mathrm{G}$ ou $\mathrm{R}$ ); $\Delta \mathrm{X}_{\text {rec }}, \Delta \mathrm{Y}_{\text {rec }}$ e $\Delta \mathrm{Z}_{\text {rec }}$ representam as correções das coordenadas aproximadas das estações $\left(X_{r e c}^{0}, Y_{r e c}^{0}\right.$ e $\left.Z_{r e c}^{0}\right)$. Designando as derivadas parciais relacionadas às coordenadas por $a=\frac{X^{S_{i}-X_{r e c}^{0}}}{(\rho)^{0}}, b=\frac{Y^{S_{i-Y_{r e c}^{0}}}}{(\rho)^{0}} \mathrm{e} c=\frac{Z^{S_{i}-Z_{r e c}^{0}}}{(\rho)^{0}}$ podem-se reescrever as Equações 16 a 19 em forma matricial: 


$$
E\{[\underbrace{\left[\begin{array}{c}
\Delta P D_{I F}^{G} \\
\Phi_{I F}^{G} \\
\Delta P D_{I F}^{R} \\
\Phi_{I F}^{R}
\end{array}\right]}_{L}\}=\underbrace{\left[\begin{array}{llllllll}
a^{G} & b^{G} & c^{G} & 1 & 0 & m_{f} & 0 & 0 \\
a^{G} & b^{G} & c^{G} & 1 & 0 & m_{f} & \lambda_{I F}^{G} & 0 \\
a^{R} & b^{R} & c^{R} & 1 & 1 & m_{f} & 0 & 0 \\
a^{R} & b^{R} & c^{R} & 1 & 1 & m_{f} & 0 & \lambda_{I F}^{R}
\end{array}\right]}_{A} \underbrace{\left[\begin{array}{c}
\Delta X_{r} \\
\Delta Y_{r} \\
\Delta Z_{r} \\
c d t_{r} \\
c d t_{s y s} \\
T z w \\
\Delta N_{I F}^{G} \\
\Delta N_{I F}^{R}
\end{array}\right]}_{x}
$$

A Matriz de Variância Covariância (MVC) das observações $\left(\Sigma_{L b}\right)$ no caso do PPP com integração GPS e GLONASS pode ser escrita da seguinte forma:

$$
\Sigma_{L b}=\left[\begin{array}{cccc}
\sigma_{P D_{I F}^{G}}^{2} & & & 0 \\
& \sigma_{\Phi_{I F}^{G}}^{2} & & \\
& & \sigma_{P D_{\mathbb{F}}^{R}}^{2} & \\
0 & & & \sigma_{\Phi \frac{R}{F}}^{2}
\end{array}\right]
$$

No caso da Equação 21, consideram-se as observações não correlacionadas. Estratégias de ponderação podem ser adotadas conforme descritas em Wang, (1999) e Silva, (2009), como por exemplo, em função do ângulo de elevação do satélite. Os experimentos e análises realizadas serão apresentados na próxima seção.

\section{Resultados e análises}

Para a realização dos experimentos e análises foram utilizados dados GNSS coletados em estações pertencentes à RBMC e distribuídas em diferentes latitudes ao longo do Brasil. A estações utilizadas, foram BOAV (Boa Vista - RR), GOJA (Jataí - GO), PPTE (Pres. Prudente SP), RECF (Recife-PE) e UFPR em Curitiba - PR, cuja localização aproximada pode ser vista na Figura 1. 


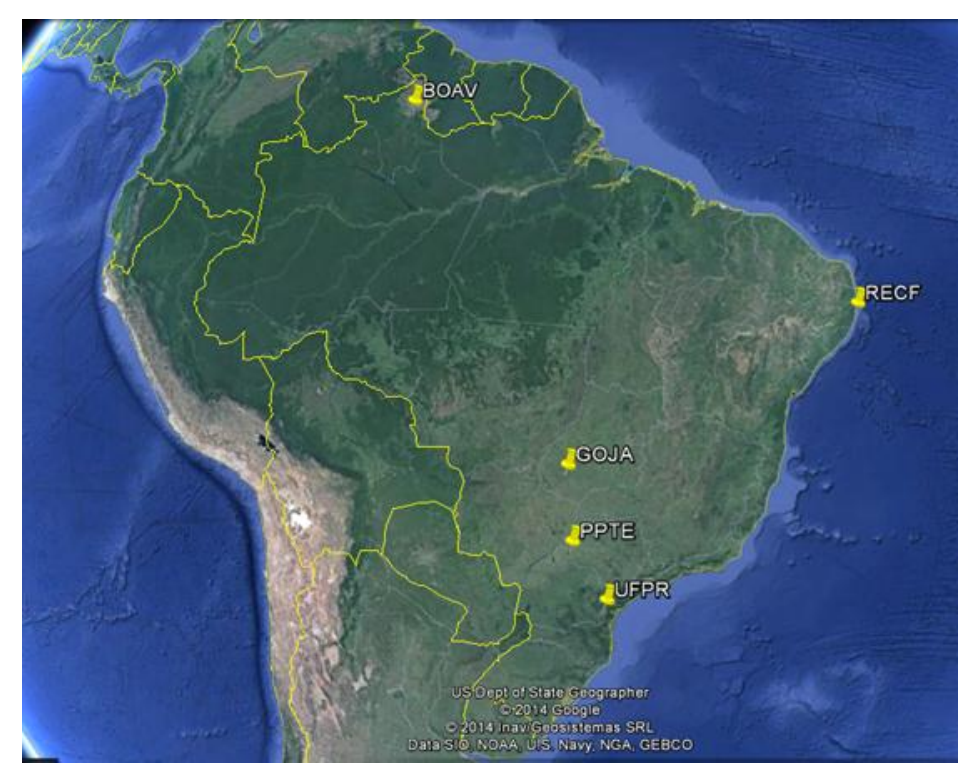

Figura 1: Localização aproximada das estações utilizadas nos experimentos.

Fonte: Google Earth.

Os dados foram processados com o software GPSPPP do NRCAN licenciado para o DECART (Departamento de Engenharia Cartografia da UFPE) para fins de pesquisa. Além disto, utilizouse o software TEQC (Translation Edition Quality Control) (UNAVCO, 2015) para a retirada de observações GLONASS do arquivo RINEX (Receiver Independent Exchange Format) com a geração de um novo arquivo. Assim primeiramente, foram processados os dados somente GPS e em seguida GPS/GLONASS.

Para a realização dos experimentos, as precisões adotadas para as medidas de pseudodistância e de fase foram, respectivamente de $1 \mathrm{~m} \mathrm{e} 0,01 \mathrm{~m}$. Além disto, foi adotado fator de variância $a$ priori unitário. Foram utilizadas órbitas precisas finais (formato .sp3) e correções finais dos relógios dos satélites (formato .clk com atualização em intervalos de $30 \mathrm{~s}$ ). Além disto, foram aplicadas correções absolutas de variação de centro de fase das antenas, carga de marés oceânicas, entre diversas outras correções disponíveis no software GPSPPP. A solução do PPP foi realizada no modo forward do ajustamento recursivo. Ao final do processamento PPP, seja usando somente dados GPS ou usando GPS/GLONASS, as coordenadas estimadas foram comparadas com as coordenadas de referência obtidas a partir da rede SIRGAS-CON (solução em ITRF 2008) com a devida atualização para a época da coleta dos dados através da solução multianual SIR1101P01 e modelos de velocidades VEMOS2009 (Velocity Model for SIRGAS 2009). Os erros em coordenadas cartesianas foram transformados para o Sistema Geodésico Local (SGL) com base nas equações apresentadas em Torge, (2001) e Seeber. (2003). Neste caso, o sistema de eixos do SGL é centrado na latitude e longitude média de todo o período de processamento e as análises foram realizadas em termos de discrepância nas coordenadas referenciadas ao SGL, ou seja, DE, DN e DU. As discrepâncias são doravante denominadas de 'erros' no SGL. A análise de acurácia foi realizada em termos de EMQ, o qual pode ser tomado como um medidor de acurácia como pode ser visto em Monico et al., (2009). Os dados das cinco estações (Figura 1) foram processados em soluções diárias com a geração de séries temporais das componentes do SGL para o ano de 2013. No caso de análise anual, a origem do sistema de eixos do SGL (DE, DN e DU) é centrada na coordenada média de todo o período de processamento para cada estação. 


\subsection{Análise de precisão e DOPs com integração GPS/GLONASS}

Considerando que se tenha disponível as coordenadas da estação e as órbitas dos satélites, podese calcular a MVC de parâmetros dada por: $\Sigma_{X A}=\hat{\sigma}_{0}^{2}\left(A^{T} P A\right)^{-1}$, em que $\hat{\sigma}_{0}^{2}$ é o fator de variância a posteriori. A matriz Jacobiana (A) e a matriz dos pesos $(\mathrm{P})$ podem ser obtidas com base nas Equações 20 e 21. Assumindo que o ajustamento seja aceito no teste Qui-quadrado, o fator de variância a posteriori pode ser considerado estatisticamente igual ao fator de variância a priori, o qual pode ser arbitrado no ajustamento. Assim, com base na MVC de parâmetros podese analisar a priori a geometria (DOP - Diluition of Precision) do GPS e GLONASS e a precisão estimadas dos parâmetros.

Para o caso deste experimento utilizou-se as coordenadas da estação RECF e a posição dos satélites foi obtida a partir das efemérides precisas considerando uma época escolhida de forma arbitrária (0h 0m $15 \mathrm{seg}$ GPS do dia 09/06/2014). Foram utilizados dados de órbita de oito satélites GPS (PRN: 01, 08, 11, 15, 19, 23, 27 e 32) e seis satélites GLONASS (Número: 02, 06, 13, 15, 18 e 24). Após a obtenção da MVC foram calculados os valores de PDOP (Positioning $D O P$ ), GDOP (Geometry DOP) e ADOP (Ambiguity DOP). No caso do posicionamento por ponto o cálculo de DOP pode ser realizado com base na MVC propagada para o SGL, cuja formulação pode ser encontrada, por exemplo, em Seeber, (2003) ou Hofmann-Wellenhof et al., (2008). No caso do ADOP quanto menor seu valor maior a probabilidade de se obter a solução inteira das ambiguidades (Teunissen et al.; 1999; Monico et al., 2014). Os valores das precisões obtidas para os parâmetros envolvidos são apresentados na Tabela 1 juntamente com o nível de melhoria em termos percentuais ao aplicar GPS/GLONASS ao invés de somente GPS.

Tabela 1: Estimativa das precisões (em metros) e melhorias percentuais

\begin{tabular}{l|l|l|l}
\hline \multirow{2}{*}{ Parâmetros } & \multicolumn{2}{c|}{ Precisões calculadas } & \multirow{2}{*}{ Melhorias (\%) } \\
\cline { 2 - 4 } & \multicolumn{1}{|c|}{ Apenas GPS } & \multicolumn{1}{c}{ GPS/GLONASS } & \\
\hline $\mathrm{X}$ & 0,696 & 0,519 & 25,44 \\
\hline $\mathrm{Y}$ & 1,066 & 0,676 & 36,57 \\
\hline $\mathrm{Z}$ & 0,662 & 0,518 & 21,67 \\
\hline $\mathrm{cdt}$ & 0,421 & 0,411 & 2,38 \\
\hline $\mathrm{cdt}_{\mathrm{xya}}$ & - & 0,711 & - \\
\hline $\mathrm{N}_{0}^{\mathrm{G}} 01$ & 6,215 & 5,978 & 3,81 \\
\hline
\end{tabular}

Verifica-se na Tabela 1 que ao utilizar dados GPS/GLONASS a melhoria percentual na precisão das coordenadas foi de aproximadamente $25 \%, 37 \%$ e $22 \%$, respectivamente para X, Y e Z. As precisões das ambiguidades GPS também apresentaram melhorias com a aplicação dos dados GPS/GLONASS sendo o máximo de aproximadamente $11 \%$ para o caso do PRN08. Os valores de PDOP, GDOP e ADOP obtidos a partir da MVC calculada para o caso apenas GPS e GPS/GLONASS são apresentados na Tabela 2, juntamente com o nível de melhorias ao usar a integração dos sistemas. 
Tabela 2: DOPs calculados e melhorias esperadas ao usar a integração

\begin{tabular}{l|l|l|l}
\hline & \multicolumn{1}{|c|}{ PDOP (m) } & \multicolumn{1}{c}{ GDOP (m) } & \multicolumn{1}{c}{ ADOP (m) } \\
\hline GPS & 1,435 & 1,496 & 6,178 \\
\hline GPS / GLONASS & 0,998 & 1,292 & 5,968 \\
\hline Melhoria (\%) & 30,453 & 13,636 & 3,399 \\
\hline
\end{tabular}

Na Tabela 2 verifica que os valores de PDOP, GDOP e ADOP ao inserir dados da constelação GLONASS juntamente com GPS apresentam, respectivamente, melhorias de aproximadamente $30 \%, 14 \%$ e $3 \%$. Com base neste experimento, pode-se dizer que o nível máximo de melhoria esperado nas precisões da estimativa das coordenadas ao inserir dados da constelação GLONASS é de aproximadamente $30 \%$. No caso das ambiguidades, o nível de melhoria obtido foi no máximo de aproximadamente $11 \%$, contudo é importante destacar que o experimento foi realizado para uma única época.

Com o objetivo de avaliar a variação diária do GDOP no PPP, os dados GPS e GLONASS coletados na estação RECF foram processados numa solução diária aplicando a estratégia de processamento usando somente dados GPS e em seguida GPS/GLONASS. A Figura 2 apresenta os valores de GDOP e número de satélites para cada um dos processamentos realizados.
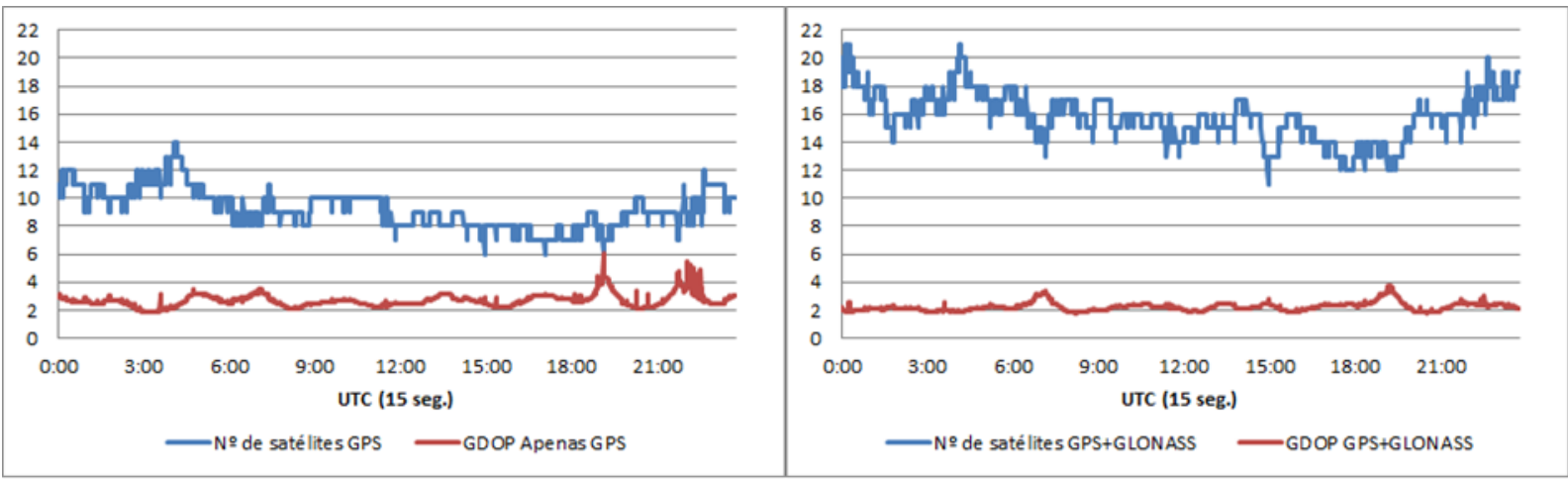

Figura 2: GDOP e n ${ }^{\circ}$ de satélites disponíveis nas soluções GPS e GPS/GLONASS

A partir da Figura 2 verifica que os valores de GDOP para o caso GPS/GLONASS apresentam, em geral, valores melhores, principalmente, para os picos próximos das 18 e 21 horas UTC onde o número de satélites GPS disponíveis foi de aproximadamente seis e atingindo 14 satélites com a inserção das medidas GLONASS.

A convergência do PPP (tempo para as precisões dos parâmetros se estabilizarem) pode ser analisada com base na série temporal das precisões estimadas das coordenadas. No caso, foram processados dados da estação RECF para cinco dias da semana GPS 1830 (Fevereiro de 2015) considerando o caso do PPP cinemático. Foram analisadas somente as primeiras quatro horas de dados (961 épocas), seja com solução GPS ou GPS/GLONASS. A série temporal das precisões resultantes (3D) neste caso pode ser vista na Figura 3. 


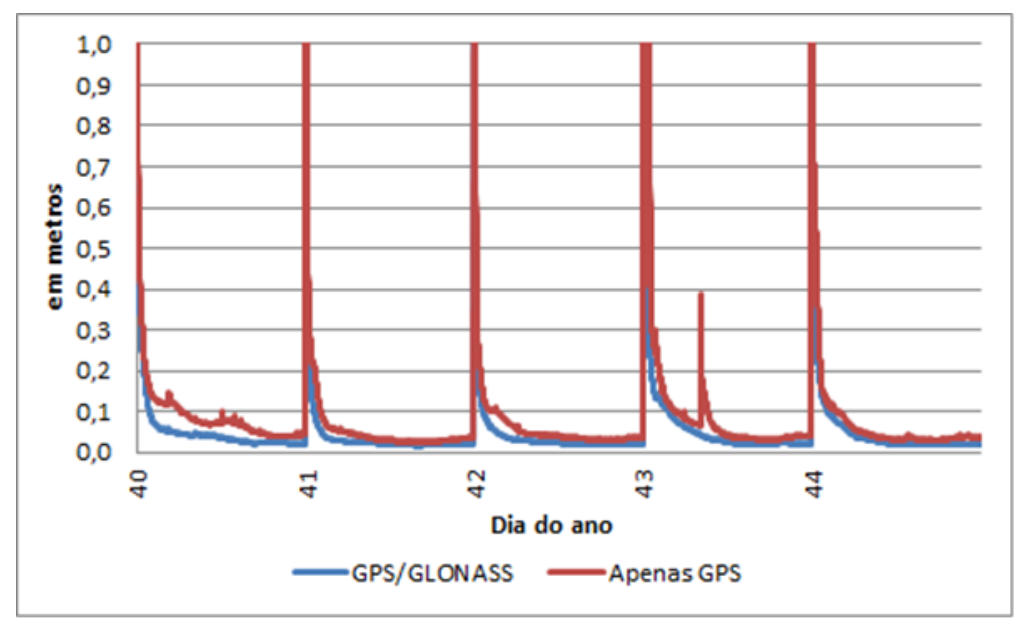

Figura 3: Precisões 3D no PPP cinemático com solução GPS e GPS/GLONASS

Verifica-se na Figura 3 que, em geral, ao aplicar a integração de dados GPS/GLONASS no PPP, as precisões das coordenadas apresentaram melhor convergência nas primeiras épocas de processamentos. Considerando os dados coletados e processados relativos às quatro primeiras horas de dados, o tempo de convergência obtido foi em média de 2 horas (480 épocas) quando utilizados apenas dados GPS, enquanto que com a utilização de dados da integração GPS/GLONASS, o tempo de convergência médio foi de 1 h e 10 min (280 épocas).

\subsection{Análise da acurácia no PPP com integração GPS/GLONASS}

Os dados GNSS das cinco estações foram processados no modo PPP estático com solução diária para o ano de 2013, cuja série temporal dos erros no SGL pode ser vista na Figura 4.

Analisando a Figura 4 verificam-se em geral valores menores nas componentes do SGL quando aplicada a integração GPS/GLONASS em relação à aplicação de somente GPS. Geralmente a componente altimétrica é a mais afetada e nota-se um comportamento cíclico ao longo do ano nas séries temporais com maiores valores próximos ao período dos solstícios, 21 de Junho (dia 172) e 21 de Dezembro (dia 355). O efeito é mais intenso para a estação BOAV, situada em Roraima, região amazônica. As altitudes das estações da referida região sofrem uma consideravelmente variação anual atingindo a ordem de centímetros devido à força exercida pelo acúmulo de água na bacia Amazônica. Este efeito sazonal também pode ser observado em estações das regiões Centro-oeste e Sudeste do País (Costa et al., 2012).

Em geral, os erros nas componentes planimétrica e altimétrica não ultrapassaram valores de 0,02 m e $0,04 \mathrm{~m}$, respectivamente. Na Figura 5 são apresentadas as séries temporais dos erros em planimetria (2D) e resultante (3D) calculados para cada uma das estações utilizadas nos experimentos. 


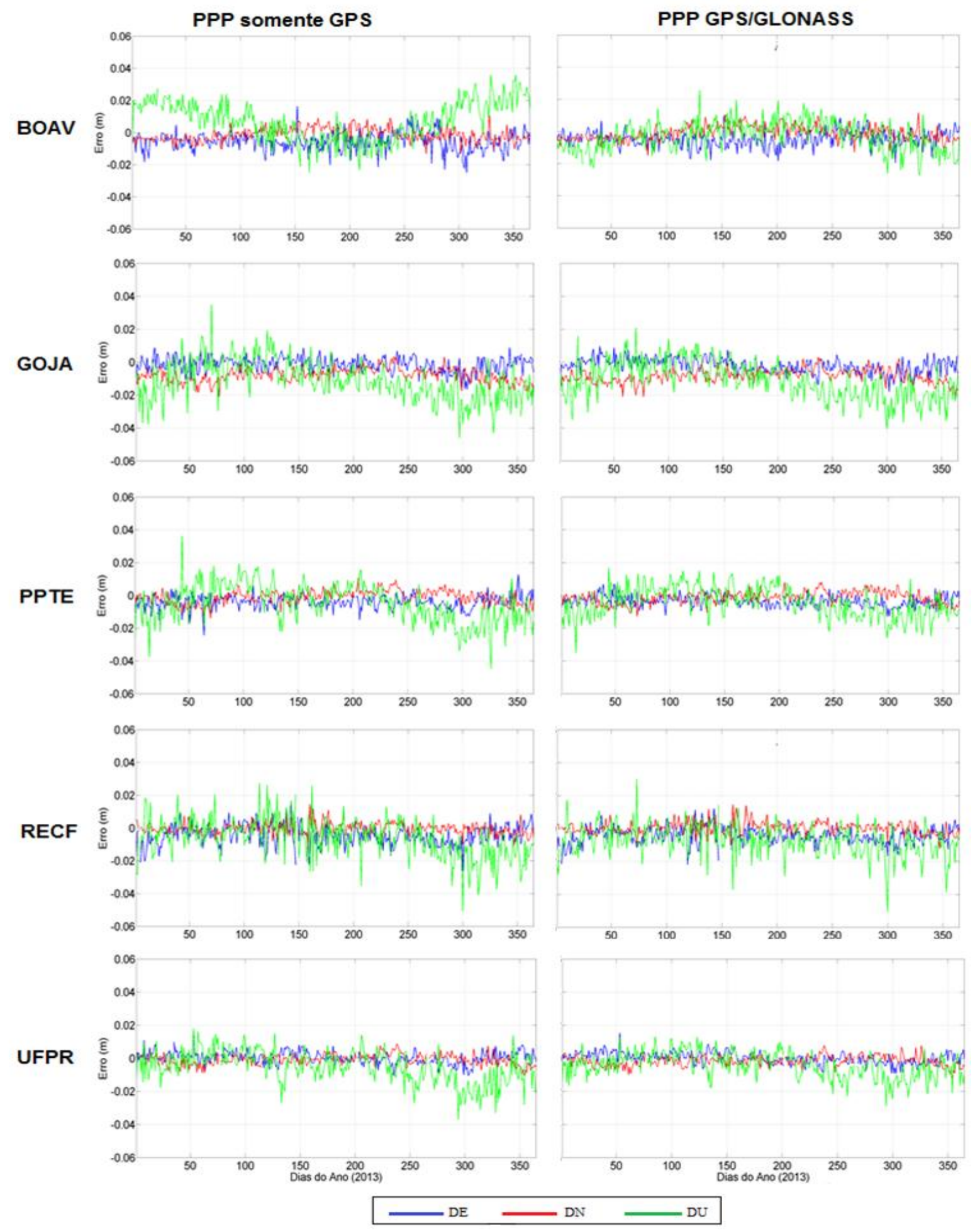

Figura 4: Discrepâncias no SGL (ano 2013) para as cinco estações analisadas 


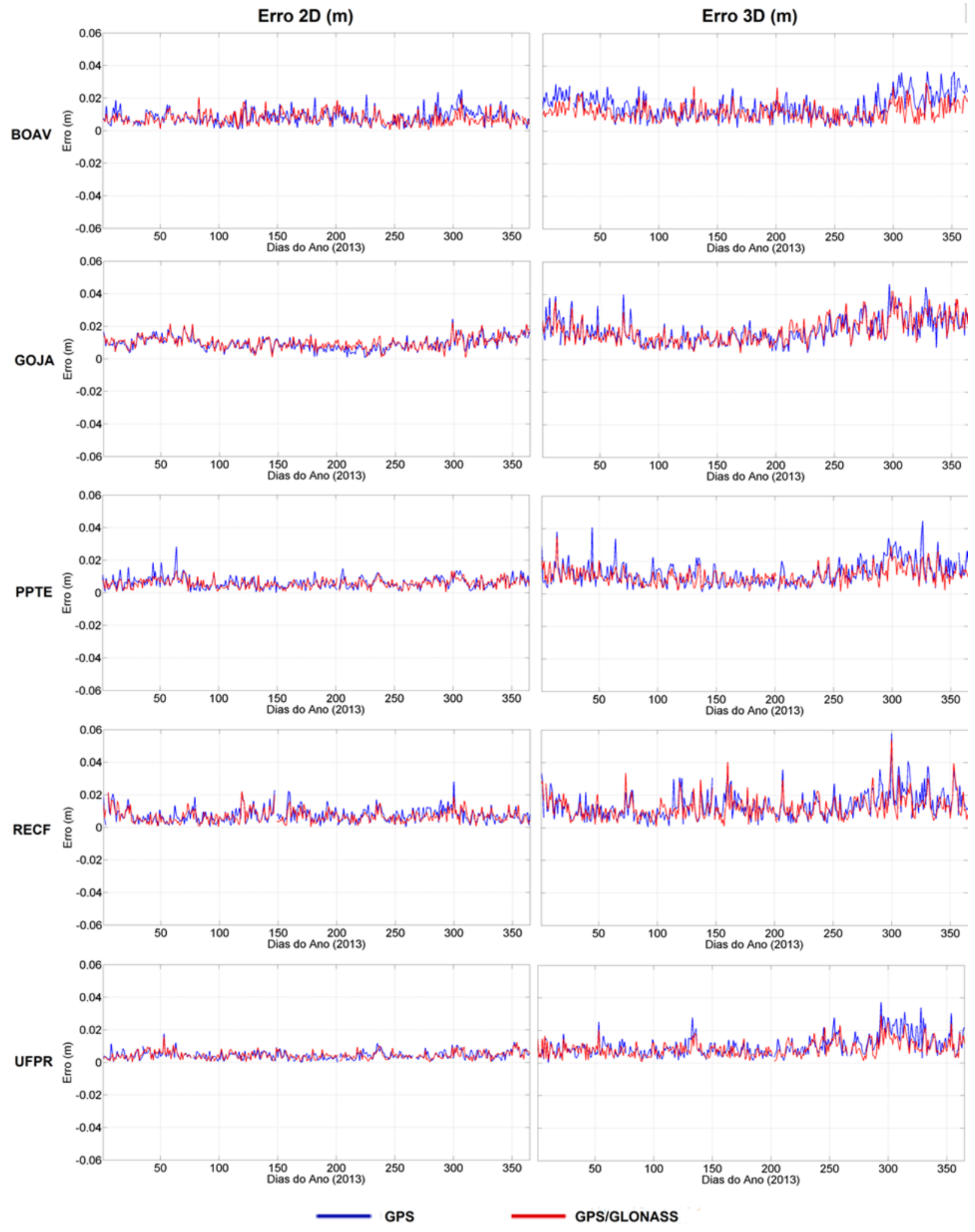

Figura 5: Erros 2D e 3D (ano 2013) para as cinco estações analisadas

A partir da Figura 5 verifica-se que os erros 2D e 3D, em geral apresentaram menores valores ao aplicar a integração GPS/GLONASS no PPP. No caso 2D, os valores máximos nas duas soluções atingem a ordem de $0,02 \mathrm{~m}$ e no caso 3D aproximadamente $0,04 \mathrm{~m}$. As diferenças nas séries temporais ao aplicar a integração em relação ao uso de somente GPS são mais aparentes para a estação BOAV. Com base nos valores diários obtidos, foi calculado o EMQ considerando 
todo o período do ano de 2013 (EMQ anual), seja para a solução PPP usando somente GPS ou GPS/GLONASS. Os valores de EMQ anual são apresentados na Tabela 3, juntamente com o nível de melhoria ao aplicar a integração GPS/GLONAS em relação ao uso de somente GPS.

Tabela 3: EMQ anual e melhorias para cada uma das estações

\begin{tabular}{|c|c|c|c|c|c|c|c|c|}
\hline \multirow{2}{*}{ Estação } & \multirow{2}{*}{ Tipo de Solução } & \multicolumn{3}{|c|}{ EMQ } & \multirow{2}{*}{$\begin{array}{c}\text { EMQ } \\
2 \mathrm{D}\end{array}$} & \multirow{2}{*}{$\begin{array}{c}\text { EMQ } \\
3 D\end{array}$} & \multirow{2}{*}{$\begin{array}{c}\text { Melhorias } \\
\text { EMQ 2D } \\
(\%)\end{array}$} & \multirow{2}{*}{$\begin{array}{c}\text { Melhorias } \\
\text { EMQ 3D } \\
(\%)\end{array}$} \\
\hline & & DE & DN & DU & & & & \\
\hline \multirow[b]{2}{*}{ BOAV } & GPS & 0,0082 & 0,0042 & 0,0139 & 0,0092 & 0,0167 & \multirow[b]{2}{*}{11,49} & \multirow[b]{2}{*}{24,47} \\
\hline & GPS/GLONASS & 0,0068 & 0,0045 & 0,0096 & 0,0082 & 0,0126 & & \\
\hline \multirow[b]{2}{*}{ GOJA } & GPS & 0,0048 & 0,0090 & 0,0164 & \begin{tabular}{|l|}
0,0102 \\
\end{tabular} & 0,0193 & \multirow[b]{2}{*}{3,52} & \multirow[b]{2}{*}{0,74} \\
\hline & GPS/GLONASS & 0,0050 & 0,0093 & 0,0160 & 0,0106 & 0,0192 & & \\
\hline \multirow[b]{2}{*}{ PPTE } & GPS & 0,0057 & 0,0042 & 0,0125 & 0,0071 & 0,0144 & \multirow[b]{2}{*}{9,56} & \multirow[b]{2}{*}{16,76} \\
\hline & GPS/GLONASS & 0,0050 & 0,0040 & 0,0101 & 0,0064 & 0,0120 & & \\
\hline \multirow[b]{2}{*}{ RECF } & GPS & 0,0077 & 0,0036 & 0,0133 & 0,0085 & 0,0158 & \multirow[b]{2}{*}{9,96} & \multirow[b]{2}{*}{10,89} \\
\hline & GPS/GLONASS & 0,0067 & 0,0037 & 0,0118 & 0,0077 & 0,0141 & & \\
\hline \multirow[b]{2}{*}{ UFPR } & GPS & 0,0037 & 0,0034 & 0,0111 & 0,0050 & 0,0122 & \multirow[b]{2}{*}{2,89} & \multirow[b]{2}{*}{17,42} \\
\hline & GPS/GLONASS & 0,0034 & 0,0035 & 0,0088 & 0,0049 & 0,0101 & & \\
\hline
\end{tabular}

Ao analisar a Tabela 3, nota-se que o EMQ anual ao aplicar a integração de dados GPS/GLONASS no PPP apresenta melhorias em relação à aplicação de somente dados GPS. A máxima melhoria em EMQ 3D foi para a estação BOAV com valor de aproximadamente $24,5 \%$ e a mínima ocorreu para a estação GOJA com valor de $0,74 \%$. No caso da estação GOJA não houve melhorias na componente planimétrica. O nível de melhoria com a componente altimétrica (EMQ 3D), para cada estação, foi aproximadamente de, 24,5\%, 0,7\%, 16,8\%, 10,9\% e 17,4\%, respectivamente para as estações BOAV, GOJA, PPTE, RECF e UFPR.

\section{Conclusões}

Este trabalho teve como principal objetivo apresentar os fundamentos matemáticos envolvidos com o ajustamento de dados no modo PPP com a integração de dados do GPS/GLONASS, além de avaliar a acurácia posicional ao aplicar a integração. Para o ajustamento dos dados no PPP, verifica-se que comparece um atraso entre os sistemas de tempo, o qual deve fazer parte dos parâmetros a serem estimados.

As análises de geometria e da precisão esperada para o caso GPS e GPS/GLONASS foram realizadas a princípio com base na MVC dos parâmetros, a qual requer o conhecimento da órbita dos satélites e da posição de uma estação para que se construa a matriz A. Verificou-se que a inserção de medidas GLONASS no posicionamento pode proporcionar melhorias na estimativa das precisões da ordem máxima de até 36\% (ver Tabela 1), haja vista que o número de satélites, geralmente, aumenta em média aproximadamente $60 \%$ por época. Ao aplicar a integração, os valores de PDOP, GDOP e ADOP ficaram melhores com valores, respectivamente de 
aproximadamente, $30 \%, 14 \%$ e 3\%, (ver Tabela 2), sendo importante destacar que se trata de análise utilizada em planejamento de coletas de dados GNSS.

Em termos de análise de acurácia do posicionamento, foram processados dados de cinco estações da RBMC distribuídas em diferentes latitudes ao longo do Brasil com solução diária no modo PPP estático e geração de séries temporais para o ano de 2013. Verificou-se um comportamento cíclico anual nas componentes do SGL em cada uma das estações, principalmente para a estação BOAV, a qual é bastante afetada pelas cargas provocadas pelo peso da massa d'água devido à cheia dos rios nos períodos de chuva que ocorrem na região. É importante destacar neste caso que ao aplicar a integração GPS/GLONASS, o efeito cíclico diminui consideravelmente, principalmente na componente altimétrica. Em relação aos erros no SGL verifica em geral valores menores nas componentes do SGL ao aplicar a integração GPS/GLONASS. No caso do EMQ 3D anual, a máxima melhoria foi para a estação BOAV com valor de $24,5 \%$ e a mínima ocorreu para a estação GOJA com valor de $0,7 \%$ e na média geral a melhoria em EMQ 3D anual foi de $14,06 \%$. Com base nos experimentos e análises realizadas, pode-se dizer que em geral, a inserção de dados GLONASS juntamente com dados GPS no PPP proporciona melhorias em termos de acurácia. A integração dos dados no ajustamento requer técnicas especificas e é necessário considerar que com a modernização de ambos os sistemas, novos sinais estarão disponíveis abrindo oportunidades de desenvolvimento e pesquisa.

\section{AGRADECIMENTOS}

Os autores agradecem ao CNPq pelo apoio financeiro em forma de projeto Universal (Processo: 475775/2013-9); a CAPES pelo apoio em forma de bolsa de mestrado junto ao Programa de PósGraduação em Ciências Geodésicas e Tecnologias de Geoinformação da UFPE; ao projeto P\&D UFPE/UNESP/EMBRAER (Processo: VDT0044-12) e ao projeto P\&D ANEEL/CHESF/UFPE (92.2011.5510.00).

\section{REFERÊNCIAS BIBLIOGRÁFICAS}

Cai, Changseng and Gao, Yang. "Precise Point Positioning Using Combined GPS and GLONASS Observations." Journal of Global Positioning System 6, (2007): 13-22.

Costa, Sonia M. A. with Silva, Alberto L., Lima, Marco A. A. and Júnior, Newton J. M. "Centro de análise SIRGAS - IBGE: Novas estratégias de processamento e combinação, e influencia da mudança de referencial global nos resultados." Boletim de Ciências Geodésicas, 18, no 1 (2012): 65-83.

Gemael, Camilo. Introdução ao ajustamento de observações: Aplicações Geodésicas. Curitiba: Editora UFPR, 1994.

Hofmann-Wellenhof, Bernhard with Lichtenegger, H. and Wasle, E. GNSS: Global Navigation Satellite Systems. Springer Wien New York: 2008.

ICD-GLONASS - Interface Control Document: Navigation radiosignal in bands L1, L2. Edition 5.1. 2008. Moscow.

Leick, Alfred. “GPS satellite surveying”. New York: John Wiley \& Sons, 2004. 
Marques, Haroldo. A. with Monico João F. G., Shimabukuro, Milton H., Oyama, R. T. and Wentz, Jens P. "PPP Em Tempo Real: Fundamentos, Implementação Computacional e Análises de Resultados no Modo Estático e Cinemático." Revista Brasileira de Cartografia 66, no 6 (2014): 1331-45.

Marques, Haroldo. A. with Monico, João F. G. and Aquino, M. "RINEX_HO: Second and thirdorder ionospheric corrections for RINEX observation files." GPS Solutions 15, (2011): 305-314.

Monico, João F. G. Posicionamento pelo GNSS: descrição, fundamentos e aplicações. São Paulo: Unesp, 2008.

Monico, João F. G. with Póz, Aluir P. D., Galo, Maurício, Santos, Marcelo C. and Oliveira, Leonardo C. "Acurácia e Precisão: Revendo os Conceitos de Forma Acurada." Boletim de Ciências Geodésicas 15, no 3 (2009): 469-83.

Monico, João F. G. with Rofatto, Vinícius F. and Mendonça, Marco A. M. "Introdução e aplicação diluição da precisão das ambiguidades GNSS - ADOP”. Boletim de Ciências Geodésicas, 20 no 2 (2014): 279-99.

Rossbach, U. Positioning and Navigation Using the Russian Satellite System GLONASS. PhD Thesis. 2001. Universität der Bundeswehr München, Neubiberg.

Sapucci, Luíz. F. "Estimativa do vapor d'água atmosférico e avaliação da modelagem do atraso zenital troposférico utilizando GPS”. diss., Universidade Estadual Paulista, 2001.

Seeber, Gunter. Satellite geodesy: foundations, methods, and applications. Berlin, New York: Walter de Gruyter, 2003.

Silva. Heloísa. A. "Avaliação de modelos estocásticos no posicionamento GNSS." diss., Universidade Estadual Paulista, 2009.

Stewart, Mike and Tsakiri, Maria. "GLONASS broadcast orbit computation." GPS Solutions 2, (1998): 16-27.

Teunissen, Peter. J. G with Odijk, Dennis and De Jong, C. D. “Ambiguity Dilution of Precision: An additional tool for GPS quality control." Department of Mathematical Geodesy and Positioning. Thijsseweg, 1999.

Torge, Wolfgang. Geodesy. Berlim, New York: Walter de Gruyter, 2001.

Unavco. "Teqc - Translation Edition Quality Control.", accessed April, 2015. http://www.unavco.org/software/data-processing/teqc/teqc.html

Cptec/Inpe. "Centro de previsão de Tempo e Estudos Climáticos/Instituto Nacional de Pesquisas Espaciais" acessed July 20, 2015. http://clima1.cptec.inpe.br/estacaochuvosa/pt.html

Wang, Jinling. "Modelling and quality control for precise GPS and GLONASS satellite positioning." PhD diss., Curtin University of Technology, 1999.

Recebido em maio de 2015.

Aceito em outubro de 2015. 


\section{ERRATA}

Na página 224, onde se lia:

Tabela 1: Estimativa das precisões (em metros) e melhorias percentuais

\begin{tabular}{l|l|l|l}
\hline \multirow{2}{*}{ Parâmetros } & \multicolumn{2}{c|}{ Precisões calculadas } & \multirow{2}{*}{ Melhorias (\%) } \\
\cline { 2 - 4 } & \multicolumn{1}{|c|}{ Apenas GPS } & \multicolumn{1}{c}{ GPS/GLONASS } & \\
\hline $\mathrm{X}$ & 0,696 & 0,519 & 25,44 \\
\hline $\mathrm{Y}$ & 1,066 & 0,676 & 36,57 \\
\hline $\mathrm{Z}$ & 0,662 & 0,518 & 21,67 \\
\hline $\mathrm{cdt}$ & 0,421 & 0,411 & 2,38 \\
\hline $\mathrm{cdt}_{\mathrm{xy}}$ & - & 0,711 & - \\
\hline $\mathrm{N}_{0} 01$ & 6,215 & 5,978 & 3,81 \\
\hline
\end{tabular}

Leia-se:

Tabela 1: Estimativa das precisões (em metros) e melhorias percentuais

\begin{tabular}{l|l|l|l}
\hline \multirow{2}{*}{ Parâmetros } & \multicolumn{2}{|c|}{ Precisões calculadas } & \multirow{2}{*}{ Melhorias (\%) } \\
\cline { 2 - 3 } & Apenas GPS & GPS/GLONASS & \\
\hline $\mathrm{X}$ & 0,696 & 0,519 & 25,44 \\
\hline $\mathrm{Y}$ & 1,066 & 0,676 & 36,57 \\
\hline cdt & 0,662 & 0,518 & 21,67 \\
\hline cdtegr & 0,421 & 0,411 & 2,38 \\
\hline $\mathrm{N}^{\mathrm{G}} 01$ & - & 0,711 & - \\
\hline $\mathrm{N}^{\mathrm{G}} 08$ & 6,215 & 5,978 & 3,81 \\
\hline $\mathrm{N}^{\mathrm{G}} 11$ & 7,190 & 6,375 & 11,34 \\
\hline $\mathrm{N}^{\mathrm{G}} 15$ & 5,881 & 5,784 & 1,65 \\
\hline $\mathrm{N}^{\mathrm{G}} 19$ & 7,187 & 6,695 & 6,84 \\
\hline $\mathrm{N}^{\mathrm{G}} 23$ & 5,985 & 5,886 & 1,64 \\
\hline $\mathrm{N}^{\mathrm{G}} 27$ & 6,089 & 5,845 & 4,00 \\
\hline $\mathrm{N}^{\mathrm{G}} 32$ & 6,244 & 5,925 & 5,11 \\
\hline $\mathrm{N}^{\mathbb{R}} 02$ & 6,095 & 5,883 & 3,47 \\
\hline $\mathrm{N}^{\mathbb{R}} 06$ & - & 6,426 & - \\
\hline $\mathrm{N}^{\mathbb{R}} 13$ & - & 6,599 & - \\
\hline $\mathrm{N}^{\mathbb{R} 15}$ & - & 6,191 & - \\
\hline $\mathrm{N}^{\mathbb{R}} 18$ & - & 6,000 & - \\
\hline $\mathrm{N}^{\mathbb{R}} 24$ & - & 6,136 & - \\
\hline & & 6,200 & - \\
\hline
\end{tabular}

Boletim de Ciências Geodésicas (2016) 22(3):574 